\title{
Evaluación de los síntomas depresivos en la población pediátrica en terapia dialítica y trasplante renal
}

Hernández-Infante EG*, Barrios-Díaz FA*, Barrios-Díaz JD*, Fernández-de-Anda I*, Cabrera-Manuel AS*, García-Huizar P*, Rosas-Cabral A*, Chew-Wong A*.

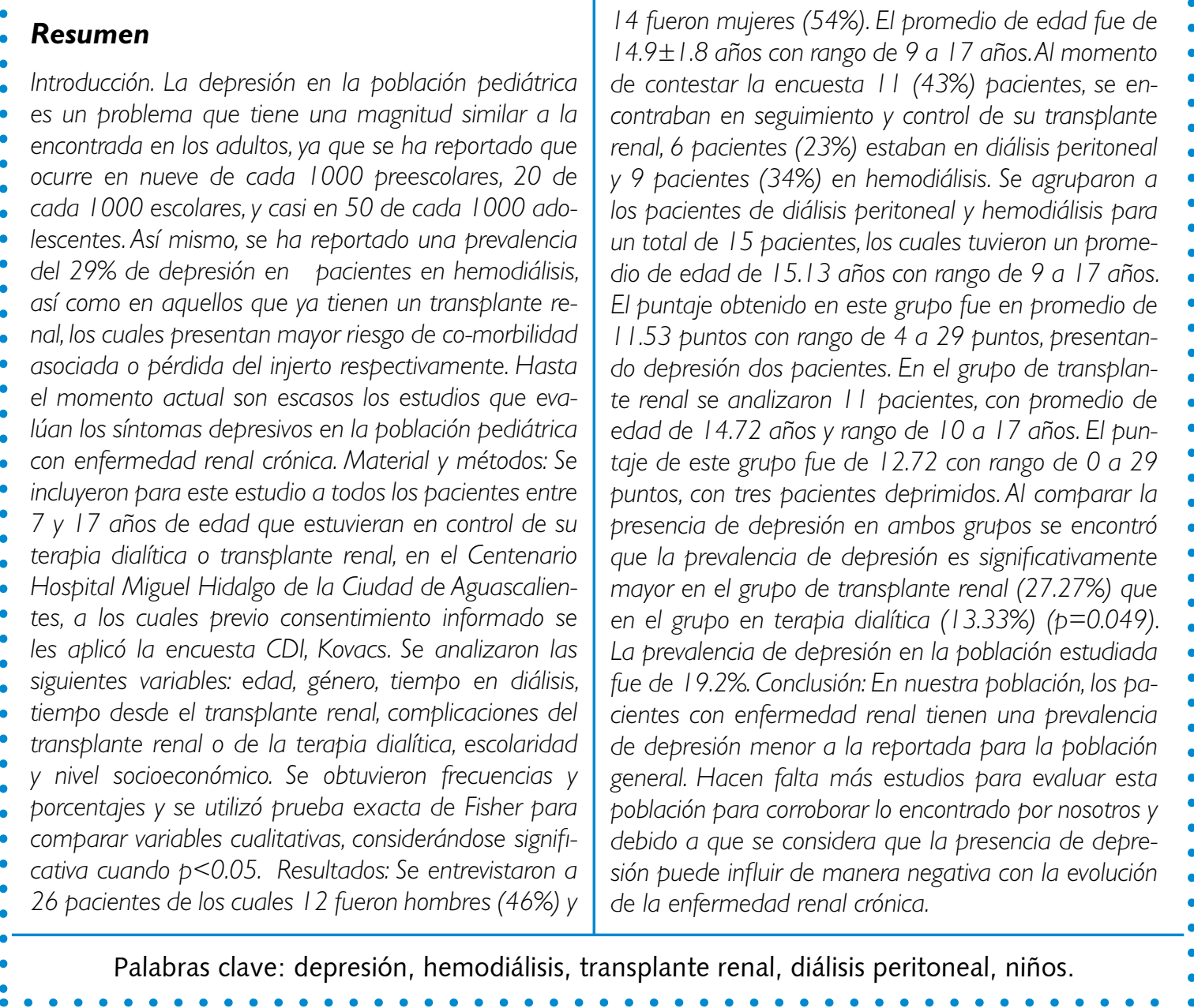

* Departamento de Medicina. Centro de Ciencias Biomédicas. Universidad Autónoma de Aguascalientes.

** Servicio de Nefrología. Centenario Hospital Miguel Hidalgo. Aguascalientes, Aguascalientes. 


\section{Introducción}

La enfermedad crónica en pediatría se define como la condición que causa un estado de enfermedad en un niño, que ha durado por más de 3 meses. Los estimados de la prevalencia de enfermedad crónica en los niños van de un 10 a un $20 \%$ y estos números aumentan significativamente (a un 30-35\%) si se incluyen los trastornos emocionales o psicosociales ${ }^{1}$.

En cuanto a la depresión, podemos decir que según el Manual Diagnóstico y Estadístico de los Trastornos Mentales de la Asociación Americana de Psiquiatría, en su cuarta edición (DSM-IV)3, la característica esencial de un episodio depresivo mayor es un período de al menos 2 semanas durante el que hay un estado de ánimo deprimido o una pérdida de interés o placer en casi todas las actividades. En los niños y adolescentes el estado de ánimo puede ser irritable en lugar de triste. El sujeto también debe experimentar al menos otros cuatro síntomas de una lista que incluye cambios de apetito o peso, del sueño y de la actividad psicomotora; falta de energía; sentimientos de infravaloración o culpa; dificultad para pensar, concentrarse o tomar decisiones, y pensamientos recurrentes de muerte o ideación, planes o intentos suicidas. Para indicar la existencia de un episodio depresivo mayor, un síntoma debe ser de nueva presentación o haber empeorado claramente si se compara con el estado del sujeto antes del episodio. Los síntomas han de mantenerse la mayor parte del día, casi cada día, durante al menos 2 semanas consecutivas. El episodio debe acompañarse de un malestar clínico significativo o de deterioro social, laboral o de otras áreas importantes de la actividad del individuo. En algunos sujetos con episodios leves la actividad puede parecer normal, pero a costa de un esfuerzo muy importante ${ }^{2}$.

Es importante la evaluación de los síntomas depresivos en la población pediátrica debido a que los niños, sobre todo en la actualidad, toman parte cada vez más activa en el conocimiento y tratamiento de las enfermedades que los afectan. Así mismo, entre sus principales intereses se encuentran los efectos de la enfermedad en su vida diaria, también les afecta el hecho de sentirse enfermos, así como las limitaciones que surgen para realizar las actividades normales ${ }^{3}$.

De esta manera se ha reportado que la depresión ocurre en aproximadamente 9 de cada 1000 preescolares, 20 de cada 1000 escolares, y casi 50 de cada 1000 adolescentes, el cual es un porcentaje ya casi similar al que se reporta en los adultos ${ }^{4}$. Esta cifra es reportada en general, para el grueso de la población, y no específica para pacientes con enfermedades agudas o crónicas.

La depresión tiene un impacto directo en la calidad de vida de los pacientes en diálisis. A su vez la depresión ya se ha identificado como un factor de riesgo para comorbilidad asociada así como para aumento el riesgo de mortalidad en pacientes que sufren depresión durante el tratamiento en diálisis ${ }^{5-7}$, así como un aumento en la 
probabilidad de perder el injerto en los pacientes que logran un trasplante ${ }^{8,9}$.

La mayoría de los estudios que han evaluado depresión en la población de enfermos renales, ya sea pacientes en diálisis peritoneal,

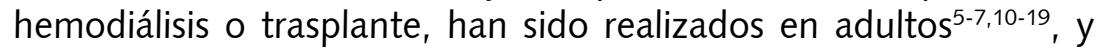
solo algunos en población pediátrica ${ }^{20,21}$.

El Inventario de Depresión para Niños (Children's Depression Inventory, CDI) ha mostrado ser útil como auxiliar clínico para el diagnóstico de la depresión en niños y adolescentes. De cualquier manera, el estándar de oro sigue siendo una entrevista clínica con el niño así como otros familiares adultos relacionados con él ${ }^{23}$. A pesar de que el CDI no es un instrumento diagnóstico, sus ítems se corresponden con los síntomas que aparecen en el DSM-IV (APA, 1995) y en el DSM-IV-TR (APA, 2000).

| | | | | | | | | | | | | | | | | | | | | | | | | | | | | | | | | | | | | | | | | | | | | | | | | | | | | | | | | | | | | | | | | | | | | | | | | | | | | | | | | | | | | | | | | | | | | | | | | | | | | | | | | | | | |

\section{Material y método}

Tomamos como población a los pacientes pediátricos de 7 a 17 años que estuvieran llevando el control de su terapia dialítica o trasplante renal en el Centenario Hospital Miguel Hidalgo. Se obtuvieron los datos para el contacto y se les invitó a participar en el estudio contestando una encuesta de 27 preguntas (CDI, Kovacs), que fue aplicada en el servicio de consulta externa, en el servicio de hemodiálisis mientras recibían su tratamiento, o en su domicilio. Esta encuesta puede obtener una puntuación de 0 hasta 2 por cada reactivo, lo que finalmente resulta en un rango de 0 hasta 54.

Posteriormente se obtuvieron del expediente clínico de cada paciente los siguientes datos:

Edad, sexo, tiempo en diálisis, tiempo desde el trasplante renal, complicaciones del trasplante renal o de la terapia dialítica, si asistían o no a la escuela, nivel socioeconómico

Se incluyeron pacientes de 7 a 17 años que estuvieran llevando a cabo el control de su tratamiento dialítico o de su trasplante renal en el Centenario Hospital Miguel Hidalgo. Una vez cumplido este criterio, y aceptando contestar el cuestionario, se entregó una hoja de consentimiento infor- mado a los padres o al adulto responsable y se les explicó en qué consistía el estudio, y debieron firmar que estaban de acuerdo con que el niño participara.

Se excluyeron a los pacientes que cumpliendo lo anterior, llevaran menos de tres meses con el diagnóstico de enfermedad renal.

Se eliminó a los pacientes que hubieran respondido al cuestionario de manera incompleta o en quienes no se encontraran los datos requeridos de consultar en el expediente clínico.

Se consideró como variable independiente la presencia de enfermedad renal crónica. En este caso estaría representada por la modalidad de terapia dialítica, ya sea diálisis peritoneal o hemodiálisis, o el trasplante renal. Se evaluó de manera cuantitativa a través el tiempo en diálisis o en trasplante renal de cada uno de los pacientes.

La variable dependiente a analizar fue la presencia de depresión, de manera cualitativa y reportamos la presencia o ausencia de dicho síntoma según lo contestado en el cuestionario.

Acerca de la herramienta utilizada para evaluar la presencia de depresión en los niños con enfermedad renal, el cuestionario 
CDI tiene una edad recomendada para su aplicación entre 7 a 17 años, y el punto de corte (percentil 90) establecido por la autora, está en la actualidad en una puntuación directa de 20, aunque se ha utilizado un punto de corte de 19 en numerosos trabajos.

Sobre la sensibilidad y especificidad del CDI para el punto de corte 19 (puntuación a partir de la cual se considera que existe presencia de sintomatología depresiva severa) en muestra española, así como otros índices, se dispone de los datos obtenidos en el estudio de la depresión infantil de Doménech y Polaino-Lorente ${ }^{24}$ sobre una $\mathrm{n}=6432$ y fueron los siguientes: sensibilidad $32.3 \%$, especificidad $94.3 \%$, mal clasificados $5.6 \%$, valor predictivo positivo $9.2 \%$, valor predictivo negativo $98.7 \%$. Se analizó la significancia de la ausencia o presencia de depresión mediante prueba exacta de Fisher.

Los estudios sobre la validez del CDI indican que es capaz de discriminar entre los niños con problemas emocionales y los niños sin problemas. Sin embargo, no diferencia a los niños deprimidos de los niños con otros trastornos.

Los datos sobre validez del CDI respecto a otros instrumentos fueron: convergencia con el Children's Depression Scale (CDS) y la 0,84 convergencia con la Escala de Evaluación de la Depresión (EED) 0,78.

Con base en esta información decidimos establecer como nuestro punto de corte el obtener 19 o más puntos en el cuestionario para considerar que un paciente presentaba sintomatología depresiva importante, en el caso de nuestro estudio.

En cuanto al análisis estadístico, las variables nominales se expresarán en porcentajes y las variables dimensionales se expresarán en media \pm desviación estándar (estadística descriptiva). El análisis inferencial se realizó con prueba de $\chi^{2}$ con corrección de Yates para variables nominales y prueba $t$ de Student para variables dimensionales. Se consideró significativo un valor de $p<0.05$.

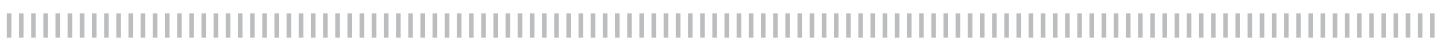

\section{Resultados}

Se logró entrevistar a un total de 26 pacientes con enfermedad renal de los cuales 12 pacientes eran hombres ( $46 \%$ ) y 14 pacientes eran mujeres (54\%). El rango de edad de los entrevistados fue de 9 a 17 años, con un promedio de edad de $14.9 \pm 1.8$ años. Al momento de contestar la encuesta, un total de 11 pacientes (43\%) estaban en seguimiento y control de su trasplante renal, 6 pacientes (23\%) se encontraban en diálisis peritoneal y 9 pacientes se encontraban en hemodiálisis (34\%).

El grupo de terapia dialítica estuvo conformado por los pacientes en las modalidades de diálisis peritoneal continua ambulatoria (DPCA) y hemodiálisis. En este grupo se incluyeron un total de 15 pacientes, en los que se observó una distribución en cuanto a género con predomino masculino,
8 pacientes (53\%), la edad osciló entre 9 y 17 años, con un promedio de edad de 15.13 años. Los puntajes obtenidos en este grupo fueron de 4 a 29 puntos, con un promedio de 11.53 puntos. Se presentaron un total de dos pacientes deprimidos, ambos en DPCA, con puntajes de 19 y 29 puntos. Dichos pacientes representan el $13.33 \%$ del total de individuos en este grupo.

En cuanto al grupo de pacientes con trasplante renal, fueron un total de $11 \mathrm{pa}$ cientes, siendo 4 hombres (42\%) y 7 mujeres $(58 \%)$. El rango de edad estuvo entre los 10 a 17 años, con una edad promedio de 14.72 años. Los puntajes obtenidos por este grupo se encontraron entre los 0 a 29 puntos, con un promedio de 12.72. En este grupo encontramos un total de 3 pacientes deprimidos con puntajes de 29, 24 y 20 en cada uno de los casos. Tabla 1. 
La prueba exacta de Fisher con la que sión resultó estadísticamente significativa se analizó la presencia o no de depre- $(p=0.049)$. Tabla 2.

\section{Tabla I}

\section{Características clínicas según modalidad de Tratamiento}

\begin{tabular}{|lccc|}
\hline & \multicolumn{2}{c|}{ Total de Pacientes $\mathrm{n}=26$ (100\%) } \\
\hline & Diálisis Peritoneal & Hemodiálisis & Trasplante Renal \\
\hline Pacientes en el grupo & $6(23 \%)$ & $9(34 \%)$ & $11(43 \%)$ \\
\hline Hombres & 4 & 4 & 4 \\
\hline Mujeres & 2 & 5 & 7 \\
\hline Rango de edad & $9-17$ & $12-17$ & $10-17$ \\
\hline Edad promedio & 15.16 & 15.11 & 14.72 \\
\hline Rango de puntajes obtenidos en CDI & $5-29$ & $4-18$ & $0-29$ \\
\hline Puntaje promedio & 14 & 9.88 & 12.72 \\
\hline Pacientes con puntaje igual o mayor a 19 & 2 & 0 & 3 \\
\hline
\end{tabular}

\section{Tabla 2}

Comparación de la presencia o ausencia de depresión en pacientes con diferentes modalidades de tratamiento sustitutivo de la función renal

\begin{tabular}{|lcc|}
\hline Variable & $\begin{array}{c}\text { Pacientes con Trasplante Renal } \\
\mathrm{N}=11\end{array}$ & $\begin{array}{c}\text { Pacientes en Diálisis } \\
\mathrm{N}=15\end{array}$ \\
\hline Edad & $14.09 \pm 2.73$ & $15.60 \pm 1.68$ \\
\hline Género (Masculino) & $42 \%$ & $53 \%$ \\
\hline Depresión & $27 \%$ & $13 \%$ \\
\hline $\mathrm{p}=0.049$ & &
\end{tabular}

Figura 1. Puntajes obtenidos por los pacientes en cada modalidad terapéutica y su relación con el tiempo de evolución del padecimiento.

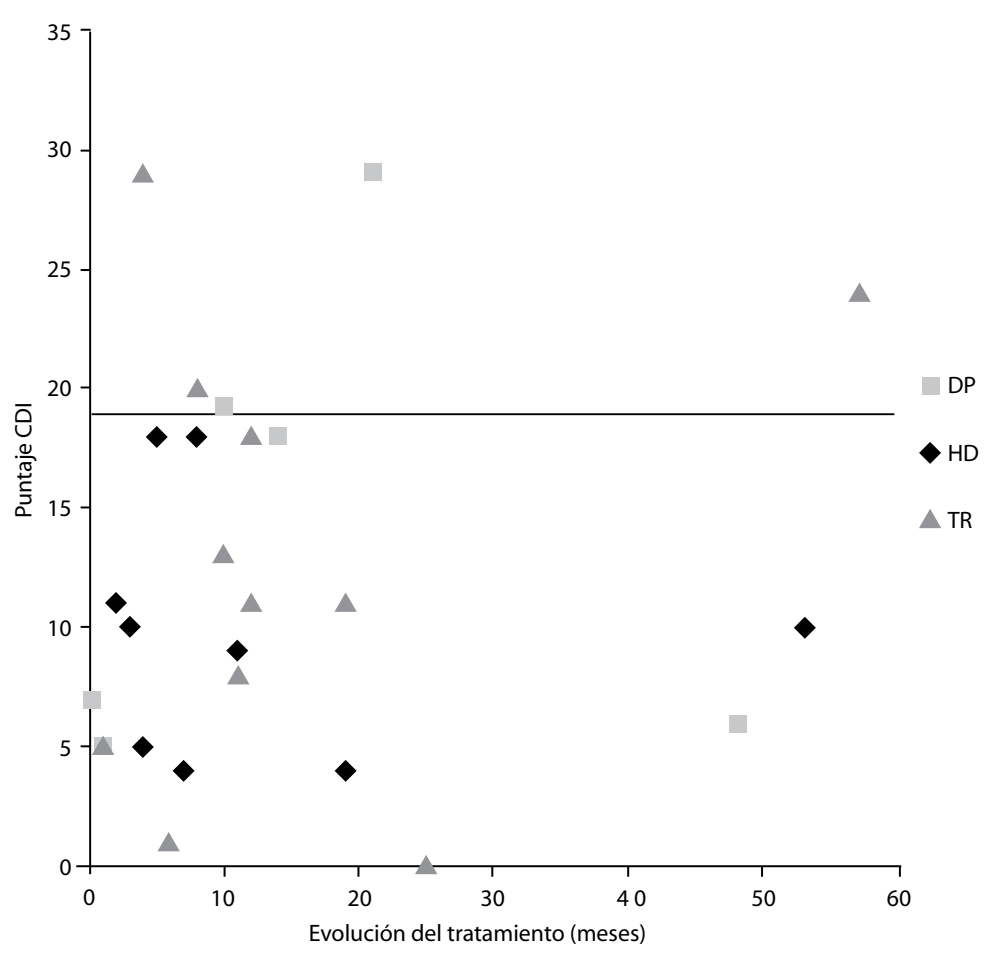




\section{Discusión}

El principal objetivo de nuestra investigación fue el determinar cuál era la prevalencia de síntomas depresivos en un grupo de pacientes con enfermedad renal y cuál es su prevalencia según lo reportado por el cuestionario CDI. A pesar de que otros trabajos $^{20}$ han reportado que no es recomendable la aplicación de instrumentos encaminados a evaluar los síntomas depresivos en enfermedades crónicas, se encontró que, en el caso especial de los pacientes con enfermedad renal crónica, se presentó una prevalencia de depresión de un 19.2\%. En un trabajo realizado por Leyva y cols. ${ }^{25}$ en el cual además de evaluar la depresión se evaluó la funcionalidad del núcleo familiar, se reportó una prevalencia de depresión del $29.8 \%$ en una población de adolescentes.

Resulta de particular interés para nuestro estudio el hecho de que la prevalencia de depresión en nuestra población (19.2\%) es menor a la prevalencia reportada en una población que presumiblemente no cuenta en su mayoría con una enfermedad crónica. Es importante resaltar que el grupo en el cual hubo más pacientes con depresión fue el de los pacientes de trasplante, siendo un $27.27 \%$ del grupo (3 pacientes) los que presentaron depresión, representando a su vez el $11.5 \%$ de la población total de nuestro estudio. Tomando en cuenta esta cantidad se encuentra que es mucho mayor que en los pacientes que conformaron el grupo de terapia dialítica, en donde sólo se encontraron dos individuos deprimidos (13.33\%) representando a su vez al 7.6\% de la población total del estudio.
La información obtenida contrasta con lo que esperábamos encontrar en un principio, debido a que aún agrupando ambas modalidades de terapia dialítica, representando una cantidad de pacientes mayor a la incluida en el grupo de pacientes de trasplante, se encontró que la prevalencia de depresión fue mayor dentro del grupo de trasplante $(27.27 \%$ ) comparado con la prevalencia encontrada dentro del grupo de pacientes en terapia dialítica (13.33\%).

Una explicación a esta situación podría encontrarse en el hecho de que uno de los tres pacientes postrasplantados renales que presentó depresión, se encontraba cursando su primer mes en terapia de trasplante, probablemente aún contando con ciertos estigmas de su terapia dialítica previa. Así mismo, un segundo paciente también en trasplante renal sufrió varios cuadros sépticos, entre otras complicaciones, lo que creemos pudo haber dejado un estigma residual en su estado de ánimo y actitud ante su enfermedad.

En relación a la prueba exacta de Fisher realizada para evaluar la presencia o no de depresión, ésta mostró significancia estadística $(p=0.049)$, a pesar de que se reunió un reducido número de pacientes. En la población mexicana ${ }^{26}$ se reporta una prevalencia de depresión del $4.5 \%$ para estudiantes adolescentes, siendo importante resaltar que se reporta una mayor prevalencia en individuos del sexo femenino, así como una mayor tendencia a presentar el padecimiento conforme avanza la edad, y conforme es menor el nivel educativo.

\section{Conclusiones}

En nuestra población, los pacientes con enfermedad renal tienen una prevalencia de depresión menor a la reportada para la población general. Hacen falta más estudios para evaluar esta población para corroborar lo encontrado por nosotros debido a que se considera que la presencia de depresión puede influir de manera negativa con la evolución de la enfermedad renal crónica. 


\section{Bibliografía}

1 Light MJ. Hazelden. Chronic Illness in Children. En: Clinician's guide to pediatric chronic illness. 1st. ed. McGrawHill 2001. P.3-7

2 Góngora-Ortega J et al. Prevalencia de enfermedad renal crónica en niños de Aguascalientes, México. Salud Pública Mex [online]. 2008, v. 50, n 6, pp. 436-437. ISSN 0036-3634.

3 DSM-IV. Manual Diagnóstico y Estadístico de los Trastornos Mentales. Asociación Americana de Psiquiatría.

4 Elliott GR, Smiga S. Depression in the child and adolescent. Pediatr Clin N Am 50 (2003) 1093-1106.

5 Drayer RA, Piraino B, Reynolds CF 3rd, Houck PR, Mazumdar S, Bernardini J, Shear MK, Rollman BL. Characteristics of depression in hemodialysis patients: symptoms, quality of life and mortality risk. Gen Hosp Psychiatry. 2006 Jul-Aug;28(4):306-12.

6 Cukor D, Peterson RA, Cohen SD, Kimmel PL. Depression in end-stage renal disease hemodialysis patients. Nat Clin Pract Nephrol. 2006 Dec;2(12):67887

7 Hedayati SS, Bosworth HB, Briley LP, Sloane RJ, Pieper CF, Kimmel PL, Szczech LA. Death or hospitalization of patients on chronic hemodialysis is associated with a physician-based diagnosis of depression. Kidney Int. 2008 Oct;74(7):930-6. Epub 2008 Jun 25.

8 Dobbels F, Van Damme-Lombaert R, Vanhaecke J, De Geest S. Growing pains: non-adherence with the immunosuppressive regimen in adolescent transplant recipients. Pediatr Transplant 2005: 9: 381-390.

9 EL-Husseini AA, Foda MA, Shokeir AA, Shehab ELDin $A B$, Sobh MA, Ghoneim MA. Determinants of graft survival in pediatric and adolescent live donor kidney transplant recipients: A single center experience. Pediatr Transplant 2005: 9: 763-769.

10 Lopes AA, Albert JM, Young EW, Satayathum S, Pisoni RL, Andreucci VE, Mapes DL, Mason NA, Fukuhara S, Wikström B, Saito A, Port FK. Screening for depression in hemodialysis patients: associations with diagnosis, treatment, and outcomes in the DOPPS. Kidney Int. 2004 Nov;66(5):2047-53.

11 Watnick S, Wang PL, Demadura T, Ganzini L. Validation of 2 Depression Screening Tools in Dialysis Patients. Am J Kidney Dis. 2005 Nov;46(5):919-24.

12 Berlim MT, Mattevi BS, Duarte AP, Thomé FS, Barros EJ, Fleck MP. Quality of life and depressive symptoms in patients with major depression and end-stage renal disease: A matched-pair study. J Psychosom Res. 2006 Nov;61(5):731-4.

13 Baines LS, Joseph JT, Jindal RM. Prospective randomized study of individual and group psychotherapy versus controls in recipients of renal transplants. Kidney Int. 2004 May;65(5):1937-42.

14 Kurella M, Luan J, Yaffe K, Chertow GM. Validation of the Kidney Disease Quality of Life (KDQOL) Cognitive Function subscale. Kidney Int. 2004 Dec;66(6):2361-7.
15 Hedayati SS, Bosworth HB, Kuchibhatla M, Kimmel $\mathrm{PL}$, Szczech LA. The predictive value of self-report scales compared with physician diagnosis of depression in hemodialysis patients. Kidney Int. 2006 May;69(9):1662-8.

16 Ross EA, Hollen TL, Fitzgerald BM. Observational study of an Arts-In-Medicine Program in an outpatient hemodialysis unit. Am J Kidney Dis. 2006 Mar;47(3):462-8.

17 Fukuhara S, Green J, Albert J, Mihara H, Pisoni R, Yamazaki S, Akiba T, Akizawa T, Asano Y, Saito A, Port F, Held P, Kurokawa K. Symptoms of depression, prescription of benzodiazepines, and the risk of death in hemodialysis patients in Japan. Kidney Int. 2006 Nov; 70(10):1866-72. Epub 2006 Oct 4.

18 Lopes AA, Bragg J, Young E, Goodkin D, Mapes D, Combe C, Piera L, Held P, Gillespie B, Port FK; Dialysis Outcomes and Practice Patterns Study (DOPPS). Depression as a predictor of mortality and hospitalization among hemodialysis patients in the United States and Europe. Kidney Int. 2002 Jul;62(1):199207.

19 Saran R, Bragg-Gresham JL, Rayner HC, Goodkin DA, Keen ML, Van Dijk PC, Kurokawa K, Piera L, Saito A, Fukuhara S, Young EW, Held PJ, Port FK. Nonadherence in hemodialysis: Associations with mortality, hospitalization, and practice patterns in the DOPPS. Kidney Int. 2003 Jul;64(1):254-62.

20 Canning EH, Kelleher K. Performance of screening tools for mental health problems in chronically ill children. Arch Pediatr Adolesc Med. 1994 Mar;148(3):272-8.

21 Berney-Martinet S, Key F, Bell L, Lépine S, Clermont MJ, Fombonne E. Psychological profile of adolescents with a kidney transplant. Pediatr Transplant. 2008 Sep 26. [Epub ahead of print]

22 Kovacs $M$. The Children's Depression, Inventory (CDI). Psychopharmacol Bull. 1985;21(4):995-8.

23 Boris NW et al. Chapter 23: Mood Disorders. En: Nelson. Textbook of Pediatrics. 17th ed. (Versión electrónica)

24 Polaino-Lorente A, Domènech E. Prevalence of Childhood Depression: Results of the First Study in Spain. J Child Psychol Psychiatry. 1993 Sep;34(6):100717.

25 Leyva-Jiménez R, Hernández-Juárez AM, Nava-Jiménez $\mathrm{G}$, López-Gaona $\mathrm{V}$. Depression in adolescents and family functioning. Rev Med Inst Mex Seguro Soc. 2007 May-Jun;45(3):225-32.

26 González-Forteza C, Jiménez-Tapia JA, Ramos-Lira $L$, Wagner FA. Application of the revised version of the Center of Epidemiological Studies Depression Scale in adolescent students from Mexico City. Salud Publica Mex. 2008 Jul-Aug;50(4):292-9. 